\title{
Viewpoint
}

\section{Considerations related to the use of molecular diagnostic tests in veterinary clinical and regulatory practice}

\author{
John R. Middleton DVM, PhD \\ Rodman G. Getchell $\mathrm{PhD}$ \\ Brian K. Flesner DVM, MS \\ Warren J. Hess DVM \\ Philip J. Johnson BVSc, MS
}

A. David Scarfe PhD, DVM

David E. Starling DVM

\begin{abstract}
From the Department of Veterinary Medicine and Surgery, College of Veterinary Medicine, University of Missouri, Columbia, MO 652II (Middleton, Flesner, Johnson); Department of Microbiology and Immunology, College of Veterinary Medicine, Cornell University, Ithaca, NY 14853 (Getchell); Division of Animal and Public Health, AVMA, Schaumburg, IL 60173 (Hess); Department of Paraclinical Sciences, University of Pretoria, Pretoria 0002, South Africa (Scarfe); Aquatic Veterinary Associates International LLC, Bartlett, IL 60103 (Scarfe); and Department of Biomedical Sciences, College of Veterinary Medicine, lowa State University, Ames, IA 500 II (Starling); and Aqueterinary Services PC, Boone, IA 50036 (Starling).
\end{abstract}

Address correspondence to Dr. Middleton (middletonjr@missouri.edu).
$\mathbf{T}$ echnological advances are creating an everwidening array of options for detecting pathogens and diagnosing diseases of concern in clinical and regulatory veterinary medicine. New technology can facilitate timelier responses and, in some instances, move assays from laboratory-based to patient-side. However, these technological advances can also create confusion in selecting the most appropriate tests and interpreting their results. The present article discusses the process of diagnostic test validation and the use of molecular diagnostic tests in clinical and regulatory veterinary medicine. For the purposes of this article, the term molecular diagnostic tests refers to tests that detect molecular components, including DNA, RNA, proteins, and metabolites, possessed or expressed by a pathogen or host as well as assays that evaluate microbial populations and their interactions and associations with a host. The main focus of the present article is assays that detect nucleic acid.

\section{Use of Diagnostic Tests in Making a Clinical Diagnosis}

Diagnostic test results, on their own, cannot be used to establish a clinical diagnosis, and they must always be interpreted in conjunction with other findings. In general, a differential list of potential diagnoses should be established on the basis of the clinical history and physical examination findings. This list of differential diagnoses is used to determine the most appropriate diagnostic tests, and results of those diagnostic tests are then used to determine the best or most likely diagnosis. Most disease conditions are not

\section{ABBREVIATIONS}

EqPV-H Equine parvovirus-hepatitis

NPHV Non-primate hepacivirus

PARR PCR assay for antigen receptor rearrangement associated with pathognomonic signs; therefore, diagnostic testing is essential. However, test results and the correct way to interpret those results are directly dependent on various aspects of the quality of the tests themselves. Therefore, particularly for molecular diagnostic tests, understanding what the tests are designed to detect, how certain we are that they are detecting what they are designed to detect, and how to incorporate the results into the overall diagnostic process is vitally important.

\section{Laboratory Quality Standards}

Before considering the quality of a diagnostic test, we should first consider the quality standards for the laboratory conducting the test. Diagnostic laboratories are expected to operate in line with standards set by national and international regulatory bodies such as the American Association of Veterinary Laboratory Diagnosticians, ${ }^{1}$ World Organisation for Animal Health (OIE) ${ }^{2}$ and International Organization for Standardization. The process for accreditation of diagnostic laboratories is beyond the scope of this article, but further information can be found on the website of the American Association of Veterinary Laboratory Diagnosticians. ${ }^{1}$

\section{Diagnostic Assay Validation}

Validation of a diagnostic assay is the process of determining that an assay has been properly developed, optimized, and standardized so that it is fit for its intended purpose. ${ }^{3,4}$ The standard process for diagnostic assay validation is well defined by regulatory agencies responsible for animal disease control programs and allows clinicians and regulators to make reasoned scientific decisions from the results regarding the presence or absence of specific pathogens or the diseases they cause. 
A detailed overview of the assay validation process is beyond the scope of the present article. However, information supporting an assay's validation process is available through the laboratory conducting the assay and includes the following factors: fitness for purpose, optimization, standardization, repeatability, robustness, analytical sensitivity and specificity, threshold level, diagnostic sensitivity and specificity, reproducibility, and ruggedness. In short, the quality of assay validation supports the quality of the assay results, which support the quality of the conclusions drawn from those results. National and state regulations require accredited veterinarians to use assays in accordance with officially recognized standards for animal evaluation, sample collection, maintaining a chain of custody for submitted samples, selecting an appropriate, accredited laboratory, and interpreting assay results when completing a certificate of veterinary inspection. Failure to adhere to these standards creates excess liability and may potentially result in loss of USDA accreditation and even loss of licensing to practice veterinary medicine.

\section{Sample type and collection}

Obtaining the correct type of sample in the correct way is paramount in obtaining useful and meaningful assay results. Sample type, collection method, handling and processing procedures, and storage method can all impact the final diagnosis. The type and number of samples required will depend on the intended purpose of the diagnostic assay and will vary depending on whether the purpose is to demonstrate freedom from infection in a population or an individual, eradicate a disease agent, diagnose clinical disease in an individual animal, or estimate the prevalence of disease in a population. Diagnostic sampling goals may differ from research goals, for which pathogen speciation, virulence testing, and assessment of host-pathogen interactions may define why and how certain samples are collected. Sample collection, handling, processing, and storage all constitute potential sources of assay variability ${ }^{5}$ and therefore must be carefully controlled.

\section{Laboratory consultation}

Laboratory diagnosticians can advise on the best assay to request (eg, cell culture, PCR assay, or isolation and identification), the type of samples to collect in any given clinical situation (eg, blood, urine, or lesion), and the number of samples to collect (eg, single or multiple). They can also advise on the pros and cons of various assays as well as the limitations of specific assays when interpreting results (eg, effect of age on pathogen prevalence). Guidance can typically be given on the most appropriate locations for sample collection, potential pathogen reservoirs (eg, host, water, or biofilm), and alternative sample types that will likely provide good results (eg, specific organs, all viscera, or whole animal). For example, the laboratory may recommend dragging sampling swabs through manure in poultry houses to detect Salmonella enteriditis ${ }^{6}$ or sampling the water supply biofilm for surveillance of Mycobacterium spp in a zebrafish tank system. ${ }^{7}$

In another context, aseptic collection of samples from a specific site on the host may be necessary. For example, diagnosis of an intramammary infection in a dairy cow will require an aseptically acquired milk sample from the affected cow's mammary gland. In the case of postmortem diagnosis, the person conducting the examination should have sufficient knowledge of anatomy and disease pathogenesis to select the necessary organs and lesions for specified assays.

\section{Molecular diagnostic tests and veterinary practice}

Polymerase chain reaction-based diagnostic tests are becoming commonplace in veterinary practice. Matrix-assisted laser desorption time-of-flight mass spectrometry uses detection of ribosomal proteins to provide genus and species identification of bacterial and some fungal pathogens, understand proteins associated with disease, and evaluate host responses to pathogens. Metagenomic approaches are expanding our understanding of infectious diseases and even being used to predict pathogen-host relationships that hinge on the host genome to give indications of susceptibility to acquired and hereditary diseases. These new associations and indications have broadened the grasp of disease ecology and epidemiology, although they should not alone be considered diagnostic. Accredited diagnostic laboratories can provide direction on the samples needed to optimize test information when using molecular diagnostic tests for disease detection or diagnosis.

\section{Application and Interpretation of Molecular Diagnostic Tests}

The following examples were developed to illustrate the application of molecular diagnostic tests to pathogen detection and disease diagnosis in various species and show how interpretation of test results might impact decisions about animal health both positively and negatively. These examples are intended to help veterinarians in clinical or regulatory practice understand how molecular diagnostic tests are fit for their purpose and how results should be used. Without this understanding, it is possible for misdiagnosis to occur or, on occasion, for a veterinarian to fail to meet regulatory requirements, resulting in increased liability.

\section{Aquaculture: unclear disease etiology}

Validated molecular diagnostic tests have been slowly adapted to applications in finfish aquaculture health. In many cases, more traditional diagnostic techniques (eg, ELISAs, histopathologic examination, or cell culture) are preferred over molecular diagnostic tests. However, for some pathogens of concern, 
these more traditional methods are not applicable. For example, some pathogens cannot be readily cultured in available fish cell lines. Piscine orthoreovirus is one example of a fish virus that is extremely difficult to culture, although it seems ubiquitous in farmed Atlantic salmon and native salmon species in the Pacific Northwest. ${ }^{8,9}$ Whether piscine orthoreovirus is associated with disease or death is widely debated in the literature. Several subtypes of the virus are thought to be associated with various disease conditions in finfish. For example, heart and skeletal muscle inflammation in Atlantic and Pacific salmon has been associated with piscine orthoreovirus 1, erythrocytic inclusion body syndrome in Pacific salmon has been associated with piscine orthoreovirus 2, and proliferative darkening syndrome in trout has been associated with piscine orthoreovirus 3. However, in none of these situations has the virus actually been proven to be the underlying cause of the disease. Further, genogroups within the subtypes of piscine orthoreovirus have been identified, ${ }^{10}$ and whether or how these genogroups might relate to disease causation or pathogenicity is unknown. Further, piscine orthoreovirus appears to be widespread in both diseased and nondiseased farmed and wild salmon, especially in species indigenous to the Pacific Northwest. ${ }^{11}$

Given the inability to culture piscine orthoreovirus in fish cell lines, the primary means for identifying the virus involve molecular diagnostic tests. However, because no causal link between the virus and disease or death has been proven, it is not clear how to interpret a positive molecular diagnostic test result, especially for salmon that appear to be healthy, or how to interpret a negative molecular diagnostic test result in salmon with heart and skeletal muscle inflammation or erythrocytic inclusion body syndrome. Furthermore, there are data suggesting that piscine orthoreovirus is a native, endemic pathogen of Pacific salmon. ${ }^{8,12}$ Thus, it is unclear whether detection of the virus in healthy farmed Atlantic salmon in the Pacific Northwest should be interpreted as a potential risk to native, wild salmon.

There are countless diseases for which an etiology is unknown or ill defined, and for certain complex, multifaceted diseases, a single pathogen may not be the sole cause. ${ }^{13}$ Having a complete understanding of the link between a pathogen and a disease is of paramount importance if molecular tests are to be used for diagnostic purposes or for making populationlevel management decisions.

\section{Livestock: false-positive and false-negative results}

Molecular diagnostic tests have become commonplace in the diagnosis of many diseases of livestock and offer the potential for improved accuracy and reduced times to obtaining results. ${ }^{14}$ However, the results must be placed in context. For example, in some regions of the world, bacterial intramammary infection in dairy cattle is being diagnosed with good sensitivity and specificity with a PCR assay performed on milk. That said, there are caveats to a positive result. For example, in the case of gram-negative mastitis, bacterial DNA can still be detected for as long as 2 or 3 weeks after results of bacterial culture of milk samples are no longer positive. ${ }^{15}$ In the situation of a positive molecular diagnostic test result but negative bacterial culture result, the molecular diagnostic test result would likely be considered a false-positive result, and reliance on the molecular diagnostic test result alone could lead to unnecessary intramammary antimicrobial treatment.

On the other hand, PCR assays can potentially detect bacterial DNA from organisms present in a sample at concentrations below the routine detection limit of bacterial culture. This can be useful when testing for organisms that cause contagious mastitis, such as Staphylococcus aureus and Streptococcus agalactiae, in bulk-tank milk samples, because these organisms tend to persist in the udder of cows, these organisms are generally spread from one cow to another during milking, and the dilution effect of milk from cows not infected with these organisms means that these organisms are typically present in bulk-tank milk samples at extremely low concentrations. Therefore, monitoring bulk-tank milk samples for DNA from these organisms is a reasonable method of performing herd-level surveillance for contagious mastitis pathogens, in that a positive molecular diagnostic test result indicates that at least some cows in the herd are shedding these bacteria in milk and contagious mastitis control procedures should be reviewed. Notably, some organisms may go undetected in milk samples even when using PCR assays, because the primers required for detection of all possible mastitis-causing pathogens cannot be included in any single test.

\section{Equine: defining disease etiology}

Theiler disease in horses, also known as equine serum hepatitis, is a severe or fatal complication associated with the use of equine-origin antiserum and other blood products. ${ }^{16-18}$ The condition was first described by Sir Arnold Theiler in 1918, but 100 years later, the underlying cause is still not completely clear. The fact that horses that had been in contact with affected horses but not treated with the same blood products could themselves be affected suggested that a transmissible or contagious pathogen was likely involved. ${ }^{18}$ However, traditional virus isolation methods intended to identify a virus in hepatic tissue from horses that died of Theiler disease had been unsuccessful.

In 2013, Chandriani et al, ${ }^{19}$ using PCR-based, parallel-sequencing technology with subsequent nucleic acid-based screening, identified a previously unknown and highly divergent member of the Flaviviridae family in horses that developed Theiler disease after being treated with botulism antiserum. This candidate virus was designated Theiler disease- 
associated virus and could be demonstrated in blood obtained from affected horses and in the equine antiserum product that had been administered to those horses. However, only RNA viral metagenomic analysis was performed in that study, and with additional study, Theiler disease-associated virus was no longer thought to be the cause of Theiler disease.

Subsequent use of additional PCR-based diagnostic methods (unbiased amplification and highthroughput sequencing of serum and tissue samples) has led to the identification of 3 additional candidate viruses that infect horses (another 2 RNA viruses and 1 DNA virus). ${ }^{20-22}$ Of importance, 2 of these novel viruses have been shown to cause hepatic disease. One is NPHV, also called equine hepacivirus or hepacivirus $\mathrm{A}$, and is a close homolog to the hepatitis $\mathrm{C}$ virus that affects humans ${ }^{23-25}$; the other is EqPV-H. ${ }^{22}$ Results of a prospective study ${ }^{26}$ suggested that EqPV-H virus could be identified in the blood, liver, or both of 18 horses that developed Theiler disease 4 to 12 weeks after administration of an equine-origin biologic (usually tetanus antiserum). In many of those cases, EqPV-H was also identified in the biologic that had been administered to an affected horse. Interestingly, retrospective testing of archived frozen samples (blood and antisera) from the Theiler disease outbreak in which Theiler disease-associated virus was first discovered showed that all of those samples were also positive for EqPV-H.

It is likely too soon to conclude that EqPV-H is the sole etiologic cause of Theiler disease. Moreover, there has been a report ${ }^{27}$ of both EqPV-H and NPHV being identified in horses with Theiler disease, suggesting that coinfection may play a role, at least in some cases. Infection with EqPV-H has also been demonstrated in horses with Theiler disease that did not receive an equine-origin biologic. ${ }^{28}$

Veterinarians need to inform owners about the risk of potentially fatal Theiler disease whenever there is a call for administration of equine-origin biologics, especially if antiserum is needed to provide protection against tetanus in wounded horses that have not been vaccinated or in which vaccination status is not current. Although the true cause of Theiler disease is still not clear, a PCR assay to test blood samples for EqPV-H and NPHV is available, and the USDA Center for Veterinary Biologics now requires that all licensed equine blood products must test negative for both EqPV-H and NPHV.

\section{Small animals: impact of extraneous factors}

The Cornell University Animal Health Diagnostic Center provides veterinary diagnostic testing and advises veterinary practitioners on test selection and testing strategies, interpretation of results, and disease prevention, surveillance, and control measures. Frequently, the center is asked to interpret results of a reverse-transcriptase PCR assay for canine distemper virus for dogs with clinical signs of respiratory tract disease. Because susceptible puppies may be exposed to wild-type canine distemper virus around the time initial vaccines are administered, it is not unusual for the clinical history of infected puppies to include recent administration of a modified-live canine distemper virus vaccine. Although PCR assays have been used to correctly diagnose distemper in thousands of dogs, they may produce false positive results in a subset of patients that have been recently vaccinated. ${ }^{a}$ Newer quantitative PCR assays that assess viral load may help discriminate whether a positive test result is due to the modified-live canine distemper virus vaccine strain or the wild-type canine distemper virus. ${ }^{\text {b }}$ Infected dogs typically have viral loads significantly higher than those for vaccinated dogs, but this may not be the case very early or very late in the course of infection with wild-type canine distemper virus. In some cases, only whole genome sequencing of the virus strain in the patient and comparison with the vaccine strain sequence can resolve the question. A direct fluorescent antibody test on swab specimens from external epithelial surfaces also may be a useful tool for diagnosis of distemper and could potentially avoid unnecessary euthanasia of vaccinated dogs with respiratory disease due to other causes. ${ }^{29}$

A second example involves use of a PARR as an aid for the diagnosis of lymphoma in dogs and cats. The PARR is an assay developed to detect clonality in a population of lymphocytes. The assay is focused on the variable-diversity-joining sequences in genes that code for cell surface receptors of lymphocytes. For reactive lymphocytes, the cell surface receptors would be expected to vary, depending on the pathogens to which they have been exposed. Because lymphomas and leukemias arise from a single progenitor cell, the cell surface receptors would be expected to be clonal. ${ }^{30}$ However, the ability of PARR to differentiate benign versus malignant disease is not foolproof. Benign clonal proliferations have been reported in animals with ehrlichiosis and leishmaniasis. ${ }^{31}$ In addition, performance of the PARR can vary among laboratories, with reported sensitivity ranging from $72 \%$ to $100 \%$ and reported specificity ranging from $96 \%$ to $100 \%$. Differences in assay protocols are one of the likely explanations for this variation in test performance. ${ }^{30}$ Formalin fixation, freezing, and exposure to deoxyribonucleases can also contribute to variations in assay results. For these reasons, it is important for veterinary clinicians to be critical of PARR results and to understand that PARR results should not be used alone to establish a diagnosis of lymphoma but should be interpreted in conjunction with other findings, including cytologic and histologic findings.

\section{Zoonotic disease: implications of positive results}

Careful interpretation of molecular diagnostic test findings early in the course of our understanding of a new disease is critical. Very early in the course of the COVID-19 pandemic, SARS-CoV-2 was detected by 
means of a reverse transcriptase PCR assay in samples from a dog. After conducting multiple tests over a period of days, Hong Kong's Agriculture Fisheries and Conservation Department said in a statement that the animal was found to have "a low-level of coronavirus infection (COVID-19) and it is likely to be a case of human-to-animal transmission." 32 The dog, a 17-yearold Pomeranian, was given to authorities on February 26, 2020, after its owner tested positive for the virus. The weak positive results from repeated molecular diagnostic tests performed in February and March 2020 that targeted different RNA segments were presumed to indicate that there was a small amount of viable coronavirus present in samples taken from the dog. However, on the basis of data from the World Organisation for Animal Health (OIE), ${ }^{33}$ it appears that results of virus isolation performed on samples in March 2020 were negative. Additionally, the dog had no signs of respiratory tract disease and appeared healthy while in quarantine, and although the dog died shortly after it was released from quarantine, it did not appear to die of COVID-19-related causes. The authorities in Hong Kong pointed out that being infected did not equate with being infectious and capable of spreading the virus. ${ }^{32}$ Whether the dog was truly infected remains unclear, and the available data simply demonstrate that viral nucleic acid was detected in samples from the dog.

Laboratory studies have suggested that cats are the domestic species most susceptible to SARS-CoV-2 infection, could develop clinical signs of disease following infection, and are able to transmit the virus to other cats. Ferrets also appear to be susceptible to infection and able to transmit the virus to other ferrets but are less likely to develop signs of disease. Dogs appear to be susceptible to infection but less likely than ferrets or cats to develop signs of disease. ${ }^{34}$ Clearly, additional studies are needed to understand whether and how different animals could be affected by the virus and what role, if any, domestic animals could play in the spread of the disease. All of these factors are important in interpreting a positive test result-in this context, the presence of nucleic acid-in the absence of other indicators of disease.

\section{Environmental contamination: incomplete assay validation}

Finally, it is important to know that molecular diagnostic tests can be used for more than detecting pathogens, but that the concepts of assay validation apply regardless of the purpose of the assay. Recently, an assay was developed to attempt to identify miniscule amounts of DNA from the invasive Asian carp in samples of river or lake water, with the hope that the assay could reduce the need for more traditional and resource-intensive animal-capture and identification processes. After Asian carp were detected in portions of the Illinois river that connect to Lake Michigan, a dispute quickly erupted between those wanting to close the river to commercial access to prevent the fish from reaching Lake Michigan and those who were confident that the various barriers in place would prevent such an invasion. Investigators repeatedly failed to capture live Asian carp above the barriers. Still, the attorneys general of 5 Great Lake states pursued court action ${ }^{35}$ in an attempt to force the US Army Corp of Engineers and Illinois Department of Natural Resources to physically close the access route between the Illinois River and the Great Lakes system in defense of preserving the Great Lakes ecosystem. The plaintiffs argued that results of the DNA assay indicated Asian carp were already present in the access route to Lake Michigan. The defendants argued that the assay for Asian Carp DNA in water samples was not sufficiently validated to produce reliable, repeatable, and dependable results and that closing the water access would also result in enormous commercial obstacles and economic damage. The district and appeals courts agreed that the assay results were not sufficient to support the plaintiffs' claims and did not grant the relief being sought, illustrating the importance of proper assay validation.

\section{Conclusions}

Selecting appropriate diagnostic tests-especially molecular diagnostic tests-and correctly interpreting their results requires understanding the targets of those tests and the role they play in the pathogenesis of disease (aquaculture and equine examples), the likelihood and meaning of false positive and false negative results (livestock example), the impact of extraneous factors on assay results (small animal examples), the true implications of a positive result (zoonotic disease example), and the extent to which the assay has been validated (environmental contamination example). Cautious interpretation of molecular diagnostic test results will reduce the likelihood of negative impacts on animal health. As stated by Fernandez et al, ${ }^{36}$ "[t]he mere detection of the presence of a pathogen in fish or shellfish samples from an outbreak does not necessarily imply that they are responsible for or even involved in the disease."

Decisions should not be made solely on the basis of diagnostic test results, especially in the absence of corroborating epidemiological, clinical, or gross evidence. As with any laboratory tests, molecular diagnostic tests are only a component of the decision-making process. An accurate diagnosis is made through the full evaluation and integration of signalment, history, clinical signs, and results of appropriately chosen and carefully interpreted laboratory tests.

\section{Acknowledgments}

No third-party funding or support was received in connection with the writing or publication of the manuscript. The authors declare that there were no conflicts of interest.

The authors thank Dr. Belinda Thompson (Cornell University) for her contributions to the example on false-positive PCR test results associated with canine distemper vaccination. 


\section{Footnotes}

a. Burton JH, Veir JK, Pearce L, et al. Detection of canine distemper virus RNA from blood and conjunctiva swabs collected from healthy puppies after administration of a modified live vaccine (abstr). American College of Veterinary Internal Medicine Forum, 2008;703.

b. Leutenegger CM, Crawford C, Levy J, et al. Canine distemper virus quantification by real-time PCR allows to differentiate vaccine virus interference and wild-type infection (abstr) J Vet Intern Med 2011;25:754.

\section{References}

1. American Association of Veterinary Laboratory Diagnosticians. Requirements for an accredited veterinary diagnostic laboratory, AC1, version 2018-07. Available at: www.aphis.usda.gov/ animal_health/nahln/downloads/AAVLD\%20Requirements\%20 2018.pdf. Accessed Nov 12, 2020.

2. World Organisation for Animal Health (OIE). Chapter 1.1.1 Quality management in veterinary testing laboratories. In Manual of diagnostic tests for aquatic animals, 2019. Available at: www.oie.int/fileadmin/Home/eng/Health_standards/ ahm/current/chapitre_quality_management.pdf. Accessed Nov 12, 2020.

3. World Organisation for Animal Health (OIE). Chapter 1.1.2 Principles and methods of validation of diagnostic assays for infectious diseases. In: Manual of diagnostic tests for aquatic animals, 2019. Available at: www.oie.int/fileadmin/Home/ eng/Health_standards/aahm/current/chapitre_validation_ diagnostics_assays.pdf. Accessed Nov 12, 2020.

4. World Organisation for Animal Health (OIE). Chapter 1.1.6 Principles and methods of validation of diagnostic assays for infectious diseases. In: Manual of diagnostic tests and vaccines for terrestrial animals, 2019. Available at: www.oie. int/fileadmin/Home/eng/Health_standards/tahm/1.01.06 VALIDATION.pdf. Accessed Apr 14, 2021

5. Purcell MK, Getchell RG, McClure CA, et al. Quantitative polymerase chain reaction (PCR) for detection of aquatic animal pathogens in a diagnostic laboratory setting. J Aquat Anim Health 2011;23:148-161.

6. Rolfe DL, Riemann HP, Farver TB, et al. Drag swab efficiency factors when sampling chicken manure. Avian Dis 2000;44:668-675

7. Whipps CM, Lieggi C, Wagner R. Mycobacteriosis in zebrafish colonies. ILAR J 2012;53:95-105.

8. Polinski MP, Marty GD, Snyman HN, et al. Piscine orthoreovirus demonstrates high infectivity but low virulence in Atlantic salmon of Pacific Canada. Sci Rep 2019;9:3297.

9. Polinski MP, Vendramin N, Cuenca A, et al. Piscine orthoreovirus: biology and distribution in farmed and wild fish. J Fish Dis 2020; 43:1331-1352

10. Saih A, Breyta RB, Warheit $\mathrm{K}$, et al. Genomes reveal ge netic diversity of piscine orthoreovirus in farmed and freeranging salmonids from Canada and USA. Virus Evolution 2020;6:veaa054

11. Jia B, Delphino MKVC, Awosile B, et al. Review of infectious agent occurrence in wild salmonids in British Columbia, Canada. J Fish Dis 2010;43:153-175

12. Garver KA, Johnson SC, Polinski MP, et al. Piscine orthoreovirus from western North America is transmissible to Atlantic salmon and sockeye salmon but fails to cause heart and skeletal muscle inflammation. PLoS One 2016;11:e0146229.

13. Bass D, Stentiford GD, Wang HC, et al. The pathobiome in an imal and plant diseases. Trends Ecol Evol 2019;34:996-1008.

14. Middleton JR. Molecular diagnostics. In: Middleton JR, Fox LK, Pighetti G, et al, eds. Laboratory handbook on bovine mastitis. 3rd ed. New Prague, Minn: National Mastitis Council Inc, 2017;25-31.

15. Hiitiö H, Pyörälä S, Taponen $S$, et al. Elimination of experimentally induced bovine intramammary infection assessed by multiplex real-time PCR and bacterial culture.J Dairy Sci 2018;101:5267-5276.
16. Messer NT, Johnson PJ. Idiopathic acute hepatic disease in horses: 12 cases (1982-1992). J Am Vet Med Assoc 1994;204:1934-1937.

17. Guglick MA, MacAllister CG, Ely RW, et al. Hepatic disease associated with administration of tetanus antitoxin in eight horses. J Am Vet Med Assoc 1995;206:1737-1740.

18. Aleman M, Nieto JE, Carr EA, et al. Serum hepatitis associated with commercial plasma transfusion in horses. J Vet Intern Med 2005;19:120-122.

19. Chandriani S, Skewes-Cox P, Zhong W, et al. Identification of a previously undescribed divergent virus from the Flaviviridae family in an outbreak of equine serum hepatitis. Proc Natl Acad Sci USA 2013;110:E1407-E1415.

20. Kapoor A, Simmonds P, Cullen JM, et al. Identification of pegivirus (GB virus-like virus) that infects horses. J Virol 2013;87:7185-7190

21. Divers TJ, Tennant BC, Kumar A, et al. New parvovirus associated with serum hepatitis in horses after inoculation of common biological product. Emerg Infect Dis 2018;24:303310

22. Tomlinson JE, Jager M, Struzyna A, et al. Tropism, pathology, and transmission of equine parvovirus-hepatitis. Emerg $\mathrm{Mi}$ crobes Infect 2020;9:651-663.

23. Burbelo PD, Dubovi EJ, Simmonds P, et al. Serology-enabled discovery of genetically diverse hepaciviruses in a new host. J Virol 2012;86:6171-6178.

24. Pfaender S, Cavalleri JM, Walter S, et al. Clinical course of infection and viral tissue tropism of hepatitis $\mathrm{C}$ virus-like nonprimate hepaciviruses in horses. Hepatology 2015;61:447-459.

25. Scheel TKH, Kapoor A, Nishiuchi E, et al. Characterization of nonprimate hepacivirus and construction of a functional molecular clone. Proc Natl Acad Sci USA 2015;112:2192-2197.

26. Tomlinson JE, Kapoor A, Kumar A, et al. Viral testing of 18 consecutive cases of equine serum hepatitis: a prospective study (2014-2018). J Vet Intern Med 2019;33:251-257.

27. Kopper JJ, Schott II HC, Divers TJ, et al. Theiler's disease associated with administration of tetanus antitoxin contaminated with nonprimate (equine) hepacivirus and equine parvovirus-hepatitis virus. Equine Vet Educ 2020;32:e5-e9.

28. Tomlinson JE, Tennant BC, Struzyna A, et al. Viral testing of 10 cases of Theiler's disease and 37 in-contact horses in the absence of equine biologic product administration: a prospective study (2014-2018). J Vet Intern Med 2019;33:258-265.

29. Kapil S, Neel T. Canine distemper virus antigen detection in external epithelia of recently vaccinated, sick dogs by fluorescence microscopy is a valuable prognostic indicator. J Clin Microbiol 2015;53:687-691.

30. Ehrhart EJ, Wong S, Richter K, et al. Polymerase chain reaction for antigen receptor rearrangement: benchmarking performance of a lymphoid clonality assay in diverse canine sample types. J Vet Intern Med 2019;33:1392-1402.

31. Keller SM, Vernau W, Moore PF. Clonality testing in veterinary medicine: a review with diagnostic guidelines. Vet Patbol 2016;53:711-1725.

32. Agriculture, Fisheries and Conservation Department, The Government of the Hong Kong Special Administrative Region. Press release: low-level of infection with COVID-19 in pet dog; Wednesday, March 4, 2020. Available at: www.afcd. gov.hk/english/publications/publications_press/pr2342. html. Accessed Nov 12, 2020.

33. World Organisation for Animal Health (OIE). COVID-19 portal: events in animals. Available at: www.oie.int/en/scientificexpertise/specific-information-and-recommendations/questionsand-answers-on-2019novel-coronavirus/events-in-animals/ Accessed Apr 15, 2021

34. Martina BE, Haagmans BL, Kuiken T, et al. SARS virus infection of cats and ferrets. Nature 2003;425:915

35. Michigan $v$ United States Army Corps of Eng'rs, 667 F.3d 765, 73 E.R.C. 1353 (7th Cir. 2011).

36. Fernandez L, Alvarez B, Menendez A, et al. Molecular tools for monitoring infectious diseases in aquaculture species. Dyn Biochem Process Biotechnol Mol Biol 2008;2:233-240. 